\title{
Soziale Netzwerkanalyse und Erzählschemata
}

\author{
EINE EXPLORATIVE VORSTUDIE
}

Friedrich Michael Dimpel

FAU Erlangen und TU Darmstadt

mail@dimpel.de

Keywords: Literary Network Analysis, Computational Literary Studies, Literary Annotation

\begin{abstract}
This study deals with nine texts which belong to three different narrative schemes: ,Brautwerbungsschema', ,Freier-Befreiungs-Geschichten' und ,Mahrtenehe-Erzählungen'. Each category is represented by three texts. While Literary Network Analysis can clearly distinguish texts of the ,Brautwerbungsschema' type from the other two types, there are no clear differences between ,Freier-Befreiungs-Geschichten' and ,Mahrtenehe-Erzählungen'.
\end{abstract}

\section{Literarische Netzwerkanalyse}

Die Literarische Netzwerkanalyse hatte keinen guten Start: Franco Moretti hat mit einfachen Netzwerkanalysen, die nicht gewichtet und nicht gerichtet waren, ${ }^{1}$ Shakespeare-Dramen analysiert. Von dem überraschenden Befund, dass sich die Bedeutung der Hauptfiguren nicht in den Netzwerkgraphen visualisieren lässt, hat sich Moretti dazu verleiten lassen, vorzuschlagen, konventionelle literaturwissenschaftliche Kategorien wie Hauptfigur versus Nebenfiguren oder ,runde' versus ,flache' Figur zu überdenken: „In Anbetracht der Verteilung spricht nichts für diese Dichotomien, sie macht eher eine radikale Neukonzeption der Figuren und ihrer Hierarchie erforderlich."2

Deutlich differenzierter operiert die Pionierstudie zur Netzwerkanalyse in der deutschen Literaturwissenschaft von Peer Trilcke: Trilckes gewichtete Netzwerkgraphen ermöglichen durchaus eine Unterscheidung von eher zentralen und eher peripheren Figuren. ${ }^{3}$ Auf der Grundlage von solchen Plots, die auf einer genaueren Datengrundlage beruhen, besteht keine Notwendigkeit, gängige Unterscheidungen von Hauptfiguren und Nebenfiguren ${ }^{4}$ zu verabschieden. ${ }^{5}$ Sicherlich darf es weniger darum ge-

1 Durchaus sehenden Auges, Moretti kommentiert diesen Umstand mit einem „Das kann nicht richtig sein.“ Vgl. Franco MoRETtI, Distant Reading. Aus dem Englischen übersetzt von Christine Pries, Konstanz 2016 (2013), 194.

2 Ebd., $198 f$

3 Peer TRILCKE, Social Network Analysis (SNA) als Methode einer textempirischen Literaturwissenschaft, in: Philip Ajouri /0 Katja Mellmann / Christoph Rauen, Hg., Empirie in der Literaturwissenschaft, Münster 2013 (Poetogenesis.Studien zur empirischen Anthropologie der Literatur 8), 201-247, hier 227-236.

4 Vgl. etwa Fotis JANNIDIS, Figur und Person. Beitrag zu einer historischen Narratologie, Berlin / New York 2004 (Narratologia 3), 104, der als Unterscheidungskriterium die Partizipation der Figur an Ereignissen in der erzählten Welt vorschlägt.

5 Zuletzt wurden SNA-Methoden verwendet, etwa bei Michael VAUTH, Netzwerkanalyse narrativer Informationsvermittlung in Dramen, in: Patrick Sahle, Hg., DHd 2019. Digital Humanities: multimedial \& multimodal. Konferenzabstracts, Köln 2019, 184-188, der Dramen von Heinrich von Kleist, insbesondere die „Familie Schroffenstein“ untersucht. Benjamin KRAUTTER / 
hen, überraschende Ergebnisse als ,distant reading mit einem „grellen Marktgeschrei der Innovation“6 zu vermarkten, sondern eher darum, um akkurate Daten zu kämpfen - auch wenn das mit Blick auf die Problemlage in narrativen Texten oft kein einfaches Geschäft darstellt, so dass man sich manchmal durchaus auch mit Annäherungen helfen muss.

Bei Trilcke dient die gleichzeitige Bühnenpräsenz im Drama als Indikator für soziale Interaktion und damit als Grundlage für die Netzwerkanalyse. Nun lässt sich diskutieren, ob eine akkuratere Erfassung von Figuren-Interaktionen als die gemeinsame Bühnenpräsenz im Drama möglich ist. Zumindest noch derzeit hat eine genauere Erfassung eine manuelle Annotation zur Voraussetzung, die idealerweise noch durch ein Inter-Annotator-Agreement abzusichern wäre: Wenn man etwa annotiert, welche gesprochene oder gedachte Figurenrede oder welcher Erzählerbericht von Figurenhandlungen einen Bezug auf welche andere Figuren enthält, lässt sich eine Beziehung zwischen zwei Figuren eher in einer Weise modellieren, wie sie bei sozialwissenschaftlichen Netzwerkanalysen üblich ist. ${ }^{7}$

Solche Studien erfordern aber derzeit noch einen erheblichen Annotationsaufwand, so dass bei längeren mittelhochdeutschen Erzählungen alternative Parameter gefunden werden müssen, die eine Modellierung der Figureninteraktion erlauben, zumal hier nicht auf Regieanweisungen wie im Drama der Moderne zurückgegriffen werden kann. Im Rahmen der vorliegenden Studie wird mit Figurenreferenzen operiert sowie zum einen untersucht, wie weit zwei Figurenreferenzen voneinander entfernt im Text stehen, und zum anderen, welche Figurenreferenzen in der Figurenrede welcher Figur vorkommen.

Eine Option, die Figurenreferenzen zu ermitteln, wäre die Nutzung des Stuttgarter Tools CRETAnno, das im Rahmen des Stuttgarter DH-Zentrums CRETA von Nora Ketschik und André Blessing entwickelt wurde. Mit CRETAnno ist eine teilautomatische Erkennung von Figurenreferenzen möglich. ${ }^{8}$ CRETAnno findet Figurenreferenzen in diesen Texten, also Ausdrücke, die eine Figur benennen oder auf eine Figur verweisen. Dabei werden auch Referenzen wie diu „frouwe“ einer Figur zugeordnet, Pronomina werden allerdings nicht erfasst. Zudem besteht derzeit noch ein erheblicher Aufwand mit der manuellen Korrektur der erfassten Referenzen. Wann es mit Hilfe von regelbasierten oder maschinellen Verfahren möglich wird, automatisch zu disambiguieren, ob sich eine Referenz wie „herzogin“ im „Parzival“ auf Orgeluse oder Jeschute bezieht, ist momentan noch nicht absehbar. Da jedoch Figurenrede in mittelhochdeutschen Texten derzeit ohnehin noch manuell annotiert werden muss, werden hier sowohl die Figurenreferenzen als auch die Textsegmente, die Figurenrede enthalten, in einem manuellen Annotationsverfahren erfasst, bei dem auch pronominale Referenzen berücksichtigt werden. ${ }^{9}$

Janis PAGEL, Klassifikation von Titelfiguren in deutschsprachigen Dramen und Evaluation am Beispiel von Lessings ,Emilia Galotti', in: Patrick Sahle (Hg.), DHd 2019. Digital Humanities: multimedial \& multimodal. Konferenzabstracts, Köln 2019, 160-164, zeigen, dass die Betweenness Centrality ein geeigneter Parameter ist, um Hauptfiguren von Nebenfiguren zu unterscheiden (Closeness dagegen weniger; so im mündlichen Vortrag). Thomas PRoIsL u. a., Dependenzbasierte syntaktische Komplexitätsmaße, in: Patrick Sahle, Hg., DHd 2019. Digital Humanities: multimedial \& multimodal. Konferenzabstracts, Köln 2019, 270-273, verwenden u.a. Zentralitätsmaße zur Ermittlung von Komplexität in Romanen.

6 TrILCKE, Social Network Analysis, 247.

7 Vgl. Friedrich Michael DIMPEL, Novellenschätze narratologisch auszeichnen und analysieren am Beispiel Victor von Scheffels ,Hugideo' und der sozialen Netzwerkanalyse, in: LiLi 47 (2017), 87-108, DOI:10.1007/s41244-017-0044-8, 104-108.

8 Vgl. https://www.creta.uni-stuttgart.de/tools/ (26.06.2019).

9 Mein Dank für die Annotationsarbeit gilt den studentischen Hilfskräften, die an diesem Projekt beteiligt waren: Sarah Dönges, Barbara Endl, Stefanie Gatzka und Sjard Salzmann. 


\section{Erzählschemata}

Mit einem ähnlichen Verfahren, das ebenfalls auf den Distanzen von Figuren-Referenzen im Text beruht, die mit den Verfahren der Netzwerkanalyse ausgewertet werden, haben Hettinger / Becker / Reger / Jannidis / Hotho untersucht, ob es möglich ist, eine Differenzierung von Roman-Subgenres quantitativ nachzuvollziehen - allerdings mit einem negativen Befund. ${ }^{10}$ Ich vermute, dass die Ergebnisse von netzwerkanalytischen Verfahren in hohem Maße abhängig von der linearen Entwicklung der Figurenkonstellation in Texten sein dürften. Zu bedenken ist, dass mit der gleichen Figurenkonstellation ein Drama, einen Roman oder eine Kurzerzählung gestaltet werden kann. Eventuell könnte dies auch für verschiedene Romansubgenres gelten, obwohl hier noch in stärkerem Maße als bei einer Differenzierung etwa von Drama, Roman und Kurzerzählung jeweils eher noch Figurentypen mit subgenrespezifischen Eigenheiten zu erwarten sind.

Mein Vorhaben setzt daher auf einer anderen Ebene an: Untersuchen will ich, ob sich Textabschnitte, die einem bestimmten Erzählschema ${ }^{11}$ oder Erzählkern ${ }^{12}$ folgen, mittels Netzwerkanalyse gruppieren lassen. Meine Thesen sind: (1) Befunde einer Literarischen Netzwerkanalyse könnten mit der Figurenkonstellation korrelieren. (2) In Texten bzw. Textsegmenten, die bestimmte Erzählkerne bzw. Erzählschemata repräsentieren, könnte eine spezifische Figurenkonstellation vorliegen. Wenn beide Thesen zutreffend sind, könnte die Netzwerkanalyse ein geeignetes Analyseinstrument für Erzählkerne bzw. Erzählschemata sein.

Untersucht werden je drei Vertreter des Brautwerbungsschemas (kurz: BW), des Mahrteneheschemas $(\mathrm{ME})$ und der Freier-Befreiungsgeschichte (FB). ${ }^{13}$ In der Freier-Befreiungsgeschichte wird eine alleinstehende Burgherrin von einem Freier belagert, der sowohl die Hand als auch die Herrschaft der Dame gewinnen will; sie lehnt die Verbindung jedoch ab und lässt ihre Burg - meist mehr schlecht als recht vor dem Angreifer verteidigen. Ein junger Ritter kommt als Retter dazu, er besiegt den Freier und darf die Burgherrin heiraten. Bei Mahrtenehe-Erzählungen kommt ein junger Ritter in eine Anderwelt, etwa auf eine Insel, und trifft dort eine Fee. Neben der erotischen Initiation hilft die Fee ihrem Geliebten, als Ritter erfolgreich zu sein. Ihre Bedingung für die Beziehung ist, dass der Ritter ein Tabu (Sehtabu, Namenstabu, ...) einhält; nach dem obligatorischen Tabubruch folgt das endgültige Ende der Beziehung oder ein Bewährungsweg. ${ }^{14}$ Beim Muster der gefährlichen Brautwerbung muss ein Brautwerber mit Hilfe eines Werbungshelfers den Widerstand des Brautvaters überwinden, um die Braut zu gewinnen. ${ }^{15}$ Ein Problem für eine digitale Auswertung liegt darin, dass mittelalterliche Texte nur selten ein Schema und eine Gattung in Reinform repräsentieren - häufig findet man Interferenzen und Gattungs-

10 Vgl. Lena Hettinger u. a., Genre classification on German novels, in: 26th International Workshop on Database and Expert Systems Applications (DEXA), 2015, 249-253.

11 Grundlegend zu Erzählschemata Armin Schulz, Erzähltheorie in mediävistischer Perspektive. Herausgegeben von Manuel Braun / Alexandra Dunkel / Jan-Dirk Müller, Berlin / Boston 2012, 159-191.

12 Vgl. zum Begriff Jan-Dirk MüLleR, Höfische Kompromisse. Acht Kapitel zur höfischen Epik um 1200, Tübingen $2007,22$. Während ein Erzählschema in der Regel einem ganzen Text oder doch großen Teilen zugeschrieben wird, können Erzählkerne auch als kleinere Einheiten beschrieben werden.

13 Die Freier-Befreiungsgeschichte wäre bei einer engen Verwendung des Begriffs „Erzählschema“ eher als Erzählkern einzustufen, da sie meist in einen größeren Kontext eingebettet ist und etwa als eine Einheit in episodisch organisierten Texten vorliegt.

14 Zur Mahrtenehe-Erzählung vgl. Schulz, Erzähltheorie, 214-231.

15 Zum Brautwerbungsschema vgl. Christian SCHMID-CADALBERT, Der ,Ortnit AW“ als Brautwerbungsdichtung. Ein Beitrag zum Verständnis mittelhochdeutscher Schemaliteratur, Bern 1985 (Bibliotheca Germanica 28), 80-100; ScHuLz, Erzähltheorie, 204-207. 
hybride ${ }^{16}$ : So lässt sich Gottfrieds „Tristan“ kaum nur auf das Brautwerbungsschema reduzieren; die hier untersuchten Brautwerbungs-Erzählungen gehören zudem verschiedenen Gattungen an, wobei die Gattungszuschreibungen bzw. Gattungsbegriffe selbst umstritten sind. (Eine mögliche Zuschreibung wäre: „Tristan“ - höfischer Roman, „Kudrun“ - Heldenepos, „König Rother“ - Spielmannsepik.)

Um dieser Problemlage zu begegnen - und auch aus Gründen der Annotationszeit - wurden nicht die vollständigen Texte annotiert, sondern die Passagen, die am ehesten dem jeweiligen Erzählschema zugeordnet werden können:

\begin{tabular}{|l|l|}
\hline & \\
\hline BW & König Rother 1-2966 \\
\hline BW & Tristan (8226-12614, Werbung um Isolde bis zur Hochzeitsnacht) \\
\hline BW & Kudrun $(204,1-567,4$, Werbung um Hilde) \\
\hline FB & Parzival $(4,27-58,26$, Belakane) \\
\hline FB & Parzival (179,13-223,30, Condwiramurs) \\
\hline FB & Gregorius (1838-2243) \\
\hline ME & Gauriel (36-868 + 2560-2949) \\
\hline ME & Ritter von Staufenberg 1-1192 \\
\hline ME & Melusine, ed. Müller, 13,3-49,14, 92,13-126,34 (weitgehend ohne Söhne-Handlung) \\
\hline
\end{tabular}

\section{Annotation}

Annotiert wird direkt in die jeweiligen Textdateien. Erneute Figurenreferenzen werden erst im übernächsten Vers (Prosa: innerhalb von 10 Wörtern) wieder annotiert, da Referenzen auf dieselbe Figur häufig eng beisammenstehen, ein Zugewinn für die Auswertung der Nähe von zwei Figurenreferenzen wäre durch die mehrfache Annotation jedoch marginal. Allerdings wird eine weitere Referenz auf dieselbe Figur innerhalb von zwei Versen dann annotiert, wenn eine Referenz auf eine andere Figur zwischen den beiden Referenzen auf dieselbe Figur steht. Figurengruppen wie etwa die Grafen im „König Rother" werden als Gruppe wie einzelne Figuren annotiert - dabei handelt es sich um eine Setzung; diese Entscheidung könnte auch anders getroffen werden. Dagegen werden funktional ähnliche, aber einzeln benannte Figuren wie die Werbungshelfer in der „Kudrun“ (Fruote, Wate, Morung) nicht zusammengefasst, sondern einzeln annotiert. Eine weitere Setzung betrifft Fälle, in denen teils einzelne Vertreter einer Gruppe und andererseits auch die Gruppe als Kollektiv agieren: Hier wird darauf verzichtet, das Handeln zu vereinheitlichen; im „Staufenberg“ werden ,Pfaffheit“ und Bischof einzeln erfasst. Ganz unkonkrete, singuläre Referenzen im Sinn von ,jedermann' werden nicht annotiert (sie haben ohnehin keine Aussicht, zu den zehn häufigsten Figuren bzw. Figurengruppen zu zählen, die hier untersucht werden): „Iwein“, V. 45f.: „in <Artus> liebte hof und den lîp / manec maget unde wîp [ohne Tag]“. Wenn etwa ein Pronomen im Plural auf zwei Figuren referiert, werden beide Figuren annotiert.

Bei der Figurenrede wird nur zwischen gesprochener und innerer Figurenrede differenziert, nicht jedoch nach direkter oder indirekter Rede; zudem wird die betreffende Figur erfasst. Innere Figurenrede umfasst demnach direkte innere Rede (innerer Monolog), indirekte innere Rede (Gesprächsbericht) und Bewusstseinsdarstellung (Emotionen, Wahrnehmungen wie sehen/hören und auch diffuse Be-

16 Vgl. exemplarisch Stephan Fuchs, Hybride Helden: Gwigalois und Willehalm. Beiträge zum Heldenbild und zur Poetik des Romans im frühen 13. Jahrhundert, Heidelberg 1997 (Frankfurter Beiträge zur Germanistik). 
schreibungen innerer Zustände). ${ }^{17}$ Verba dicendi werden bei direkter Rede nicht mit in die annotierte Passage eingeschlossen, bei indirekter Rede jedoch schon mit ausgezeichnet. ${ }^{18}$ Bei Figurenreden, die zwei Sprecher haben, werden in doppelter Annotation beide Sprecher erfasst und mit nachgestelltem <gr_multi>-Tag versehen, um Auswertung und Syntaxcheck zu erleichtern. Geschachtelte Figurenreden werden nur dann ausgezeichnet, wenn es sich um ,ausgewachsene“ Binnenerzählungen handelt (mehr als 10 Verse). Ansonsten wird nur die ,äußere' Rede annotiert. Spezialfälle wie sie Annelen Brunner mit Hilfe von „@non-fact“, „@border“, „@ narr“ erfasst, werden nicht annotiert. ${ }^{19}$ Fragmente einer indirekten inneren Figurenrede, die weniger als drei Wörtern umfasst, werden nicht annotiert, wenn dabei ohnehin keine Referenz auf eine andere Figur vorliegt. Nicht als innere Figurenrede annotiert würde demnach: „Er ging traurig weiter“ (fiktives Beispiel). Annotiert würde jedoch: „Er dachte traurig an Georg“ oder „Er hörte Georg“.

\section{Auswertung}

Ein erstes Perl-Skript sucht Tags und ermittelt ihre Häufigkeit, womit Tippfehler ermittelt werden können. Ein erster Syntaxcheck gleicht die öffnenden und schließenden Tags bei Redewiedergabe miteinander ab. Eliminiert werden Tags wie Rezipient, Erzähler, Teufel, Maria (als Anrufungsinstanz); zudem wird eine Namensliste für die Netzwerkanalyse mit den zehn häufigsten Figuren geschrieben.

Ein zweites Perl-Skript ermittelt für die zehn häufigsten Figuren die jeweils kürzesten Distanzen zwischen je zwei Figurenreferenzen in Worten. Da beim vorliegenden Korpus ein Normalisierungs-Problem besteht - es liegen verschieden lange Texte vor, wodurch verschieden häufige Figurenokkurrenzen bedingt sind, wird versucht, dieser Problemlage mit einer doppelt ternären Gewichtung der Distanzen zu den zehn häufigsten Figuren zu begegnen.

Die erste ternäre Gewichtung 1 beruht auf drei Schwellen (bspw. 10-20-30 Wörter). Eine Distanz zwischen zwei Figurenreferenzen, die kleiner ist als die Schwelle 1 (bspw. 10 Wörter), erhält die Gewichtung „3“. Eine Distanz, die kleiner ist als die Schwelle 2 (bspw. 20 Wörter), erhält die Gewichtung „2“. Eine Distanz, die kleiner ist als die Schwelle 3 (bspw. 30 Wörter), erhält die Gewichtung „1“.

Die zweite ternäre Gewichtung bezieht die Anzahl der nahen Referenzen von zwei Figuren ein: Wie viele Distanzen mit den Distanzgewichtungen 1, 2 oder 3 zwischen den Figuren A und B wurden gefunden? Hier werden die drei höchsten Distanzgewichtungen summiert. Wenn beispielsweise für die Figuren $A$ und $B$ zwei nahe Distanzen gefunden wurden, die einmal die Gewichtung 3 und einmal die Gewichtung 1 erhalten haben, beträgt die Gesamtgewichtung der Relation 4. Wenn beispielsweise für die Figuren $C$ und D vier nahe Distanzen gefunden wurden, die einmal die Gewichtung 3 und zweimal die Gewichtung 2 und einmal die Gewichtung 1 erhalten haben, beträgt die Gesamtgewichtung der Relation 3+2+2=7. Der Maximalwert beträgt 9. Denkbar wäre, bei diesem Verfahren noch einen Faktor zu verwenden, damit eine höhere Anzahl an Distanzgewichtungen eingehen muss, bis die Gesamtgewichtung steigt.

17 Vgl. Gert HüBNER, Erzählform im höfischen Roman. Studien zur Fokalisierung im ,Eneas', im ,Iwein' und im ,Tristan', Tübingen 2003 (Bibliotheca Germanica 44), 47.

18 In Anschluss an Annelen BRUNNER, Automatische Erkennung von Redewiedergabe. Ein Beitrag zur quantitativen Narratologie, Berlin / Boston 2015 (Narratologia 47), 66f.

19 Vgl. BRunneR, Redewiedergabe, 72ff. 
Für die zehn häufigsten Figuren wird der Degree der jeweiligen Figuren und sowie der Gesamtdegree berechnet. Der Degree ist ein Zentralitätsmaß, das aus der Anzahl der Beziehungen einer Figur besteht. Weiterhin wird der Closeness-Wert für die Figuren sowie der Durchschnitt dieser Closeness-Werte berechnet. Closeness ist ein Zentralitätsmaß, das misst, wie lang die Wege im Durchschnitt sind, über die eine Figur alle anderen Figuren im Netzwerk erreichen kann: Wie nah liegt ein Akteur an den anderen Akteuren? ${ }^{20}$ Ein Python-Networkx-Skript berechnet die Betweenness-Zentralität aller Figuren und den Mittelwert dazu und gibt eine DL-Datei der Affiliationsmatrix für den UCInet-Import aus. Betweenness ist ein Zentralitätsmaß, das misst, wie viele kurze Verbindungen zwischen 2 Akteuren über einen Akteur in der Mitte laufen. ${ }^{21}$ „Personen, die zwei ansonsten unverbundene Teilpopulationen miteinander verbinden, sind typischerweise Akteure mit hoher betweenness centrality." 22

Für die Redewiedergabe muss das Verfahren nur leicht modifiziert werden: Ein Perl-Skript sucht zu jeder Figur den Text, der von Redewiedergabe-Tags umschlossen ist, und zählt darin die Figurenreferenzen auf alle Figuren. Eine gerichtete Beziehung von Figur A zu Figur B wird dann erfasst, wenn die Redewiedergabe von Figur A eine Referenz auf Figur B enthält. Als Gewichtung wird die Anzahl der Referenzen von Figur A auf Figur B verwendet (normalisiert: geteilt durch die Anzahl der Beziehungen im Text insgesamt). Die Berechnungen erfolgen wie bei den Distanzen; hier jedoch mit Indegree und Outdegree (gewichtet): Outdegree ist ein Zentralitätsmaß, das erfasst, wie viele gerichtete Beziehungen zu anderen Figuren von Figur A ausgehen. Indegree ist ein Zentralitätsmaß, das erfasst, wie viele gerichtete Beziehungen von anderen Figuren bei Figur A eingehen.

\section{Vergleichbarkeit von Figuren und Aktanten}

Ich verwende hier Aktantenlogik nach Greimas in der pointierten Darstellung von Warning, um Figurentypen einander zuzuordnen. ${ }^{23}$ Demnach wird operiert mit einem Helden, dem Subjektaktanten, einem Opponenten, der besiegt werden muss, einer Helferfigur (Adjuvant), und einem Objektaktanten - bspw. im Brautwerbungsschema die Braut, die der Brautwerber gerne erwerben möchte. Eine Zuordnung ist nicht immer ganz unproblematisch: In der „Kudrun“ handeln die Werbungshelfer mehr als der Brautwerber, so dass man auch überlegen könnte, ob Hetel tatsächlich Subjektaktant ist. In MahrteneheErzählungen ist die Bestimmung des Opponenten nicht unproblematisch: Wohl wichtiger als Zweikampf-Gegner sind die Figuren, die den Helden zum Tabubruch verführen, doch dabei handelt es ich meist um Freunde oder enge Verwandte, also gerade nicht um die klassischen Bösewichte. Die Position des Adjuvanten ist in Mahrtenehe-Erzählungen nicht unbedingt besetzt oder identisch mit der weiblichen Hauptfigur.

20 Vgl. Mark Trappmann / Hans J. Hummell / Wolfgang Sodeur, Strukturanalyse sozialer Netzwerke. Konzepte, Modelle, Methoden, Wiesbaden 2005, 46-51.

21 Vgl. Dorothea JANSEN, Einführung in die Netzwerkanalyse, Opladen 2003 (UTB 2241), $141 \mathrm{f}$.

22 Trappmann / Hummel / Sodeur, Strukturanalyse sozialer Netzwerke, 52.

23 Algirdas Julien GreimAs, Strukturale Semantik. Methodologische Untersuchungen. Autorisierte Übersetzung aus dem Französischen von Jens Ihwe, Braunschweig [1966] 1971 (Wissenschaftstheorie, Wissenschaft und Philosophie 4); Rainer WARNING: Formen narrativer Identitätskonstitution im höfischen Roman, in: Odo Marquard / Karlheinz Stierle, Hg.: Identität, München 1979 (Poetik und Hermeneutik 8), 553-589. 


\section{Netzwerkgraphen und Zentralitätsmaße}

Die Netzwerkgraphen wurden mit UClnet erstellt. ${ }^{24}$ Kanten und Knoten sind nach Betweenness gewichtet. Die Brautwerbungs-Erzählungen sind recht homogen. Der ,Gauriel' ähnelt etwas den Brautwerbungs-Erzählungen, er ist jedoch nicht ganz so stark verbunden. Eine klare Differenzierung zwischen Mahrtenehe-Erzählungen und Freier-Befreiungs-Erzählungen ist nicht erkennbar. Die Differenzierung zwischen Haupt- und Nebenfiguren beim „Gregorius“ ist besonders deutlich ausgeprägt.

Bei Redewiedergabe wird erfasst, welche Figur auf welche andere Figur Bezug nimmt, es handelt sich also um gerichtete Beziehungen. Rot sind einseitige Beziehungen dargestellt (Figur A erwähnt Figur B, aber nicht umgekehrt), blau sind gegenseitige Beziehungen dargestellt.

Auch hier sind die Brautwerbungs-Erzählungen auffällig homogen: Sie sind wieder stark verbunden, es gibt multiple Bezugnahmen. Im "Gregorius" ist der Textanteil, der als Redewiedergabe vorliegt, recht niedrig. Hier deutet sich ein Problem an: Das gleiche Erzählschema kann mit mehr oder weniger Figurenrede erzählt werden. In welchem Umfang Figuren andere Figuren in Figurenrede erwähnen, muss nicht zwingend mit dem Schema zusammenhängen. Denkbar wäre immerhin, dass es gewisse Präferenzen bei bestimmten Erzählmodellen geben könnte.

Wiederum differieren Mahrtenehe-Erzählungen und Freier-Befreiungs-Erzählungen schwächer als Brautwerbungs-Erzählungen. Die Plots ermöglichen viele interessante Einzelbeobachtungen, die aus Raumgründen hier nicht ausgebaut werden können. Exemplarisch sei auf die zahlreichen einseitigen Beziehungen in den Mahrtenehe-Erzählungen verwiesen; die Beziehung zwischen Hauptfigur und Fee sticht hier heraus. Weiterhin ist in der „Kudrun“ die Braut Hilde stark zu den Werbungshelfern hin orientiert - etwa mehr zum Werbungshelfer Horand als zum künftigen Ehemann Hetel.

\section{Distanzen 10-20-30}

Freier-Befreiung: Greg, Pz_Bela, Pz_CWA
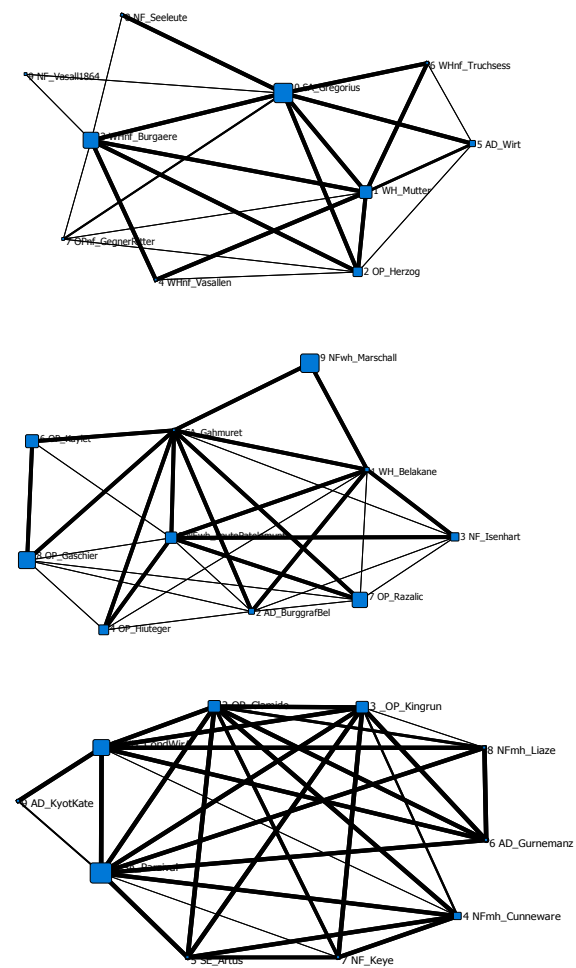

Brautwerbung: Kudr, Roth, Trist
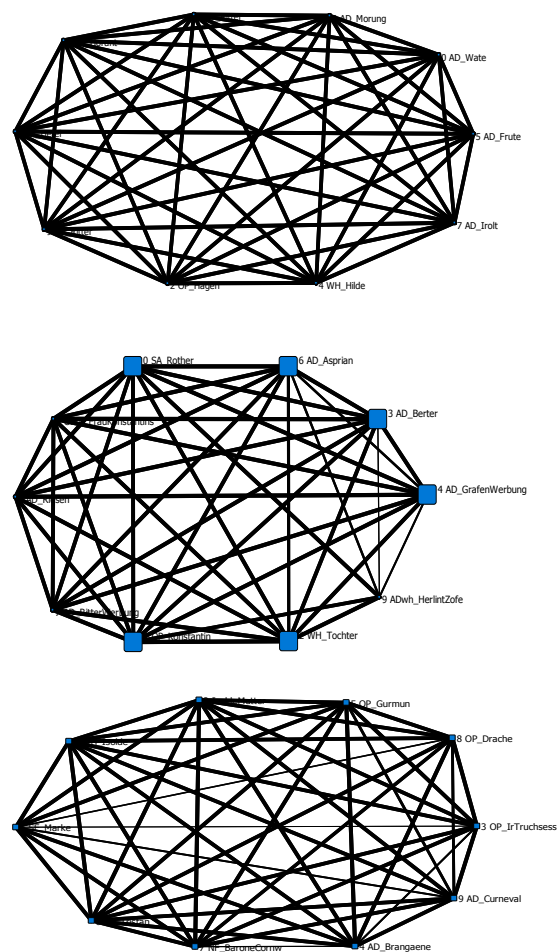

Mahrtenehe: Gau, Mel, Stau
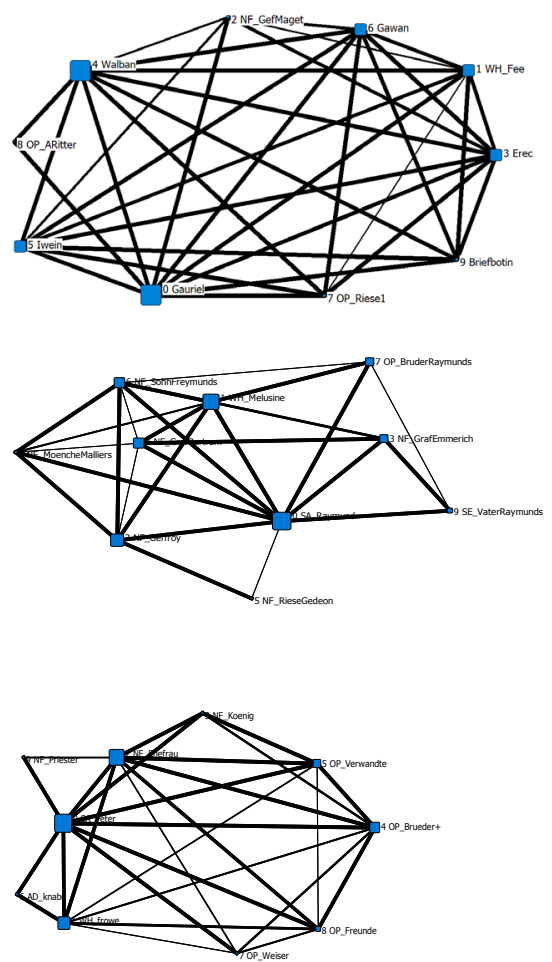

24 S.P. Borgatti / M.G. Everett / L.C. Freeman, Ucinet 6 for Windows: Software for Social Network AnalysiHarvard, MA: Analytic Technologies 2002. 
Freier-Befreiung: Greg, Pz Bela, Pz CWA
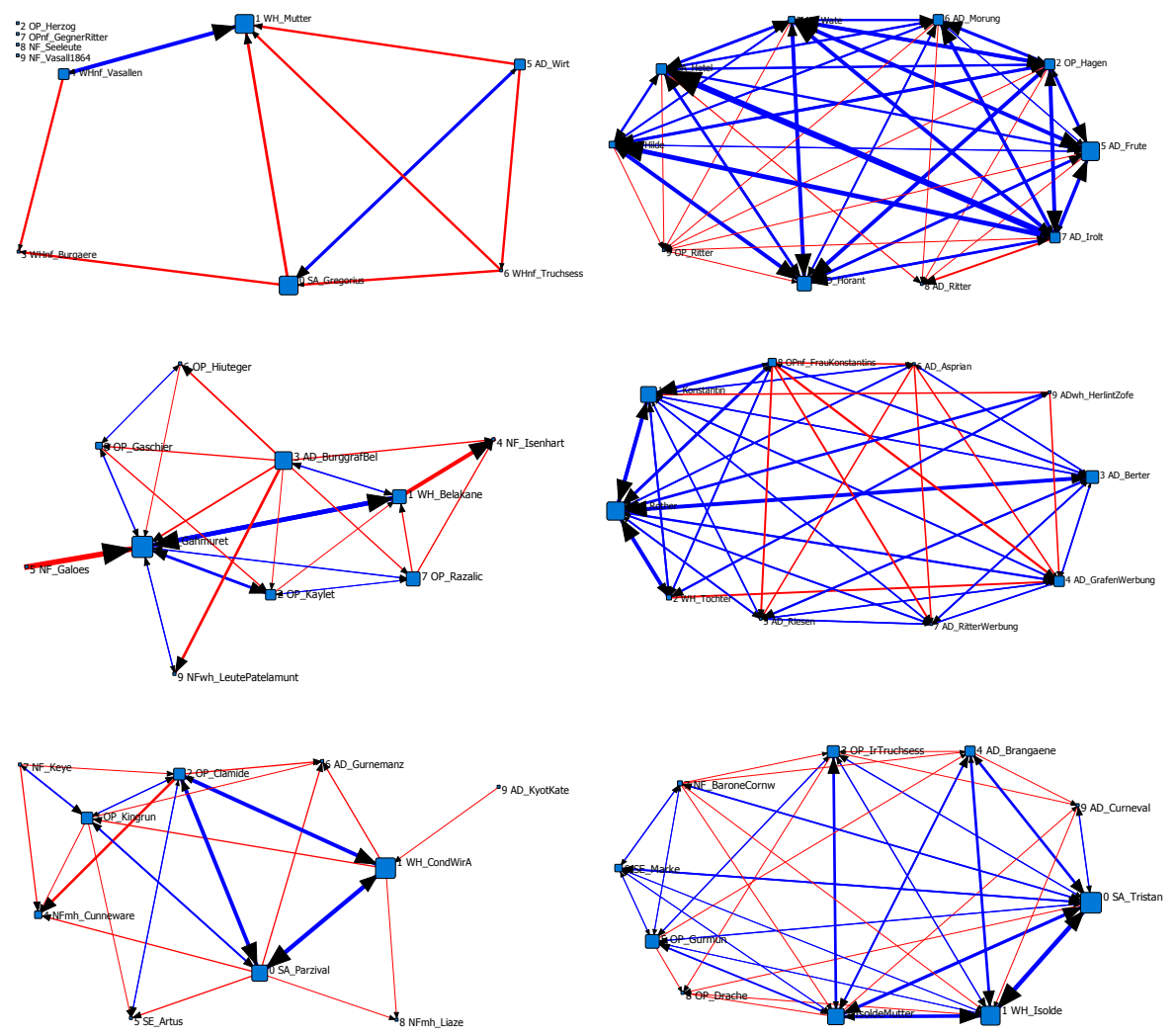

Mahrtenehe: Gau, Mel, Stau
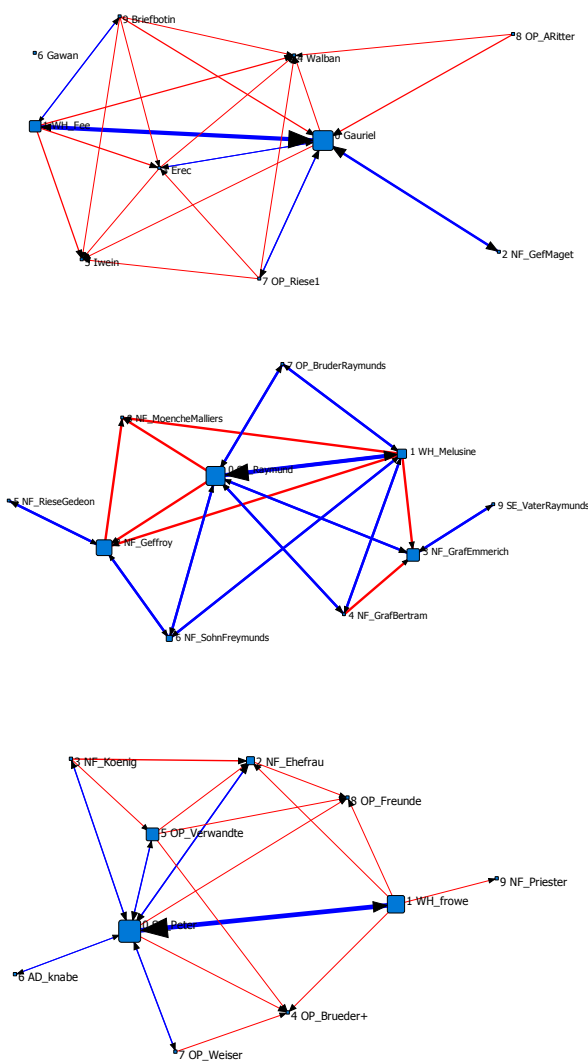

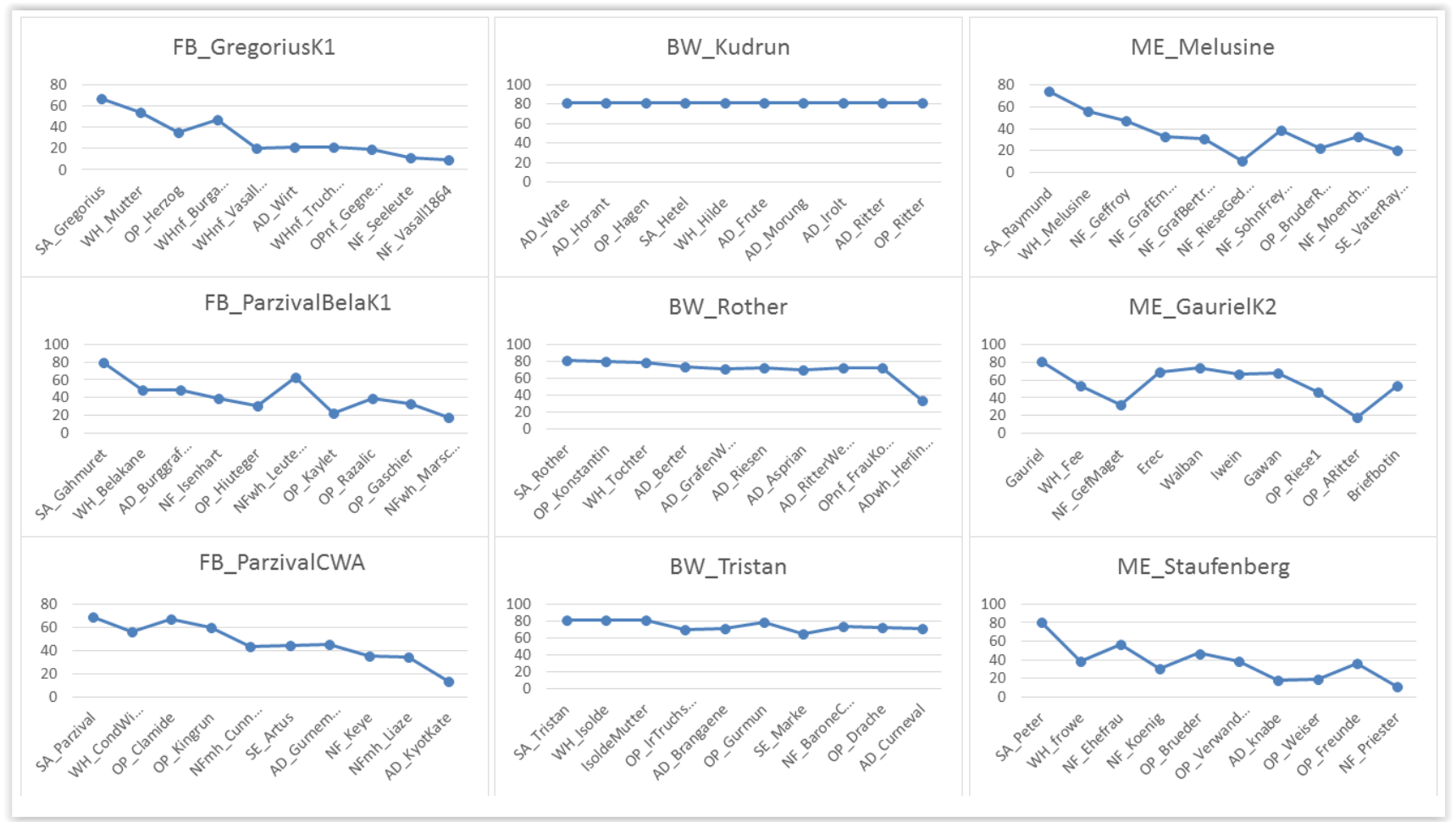

Distanzen: Degree je Text für die zehn häufigsten Figuren 


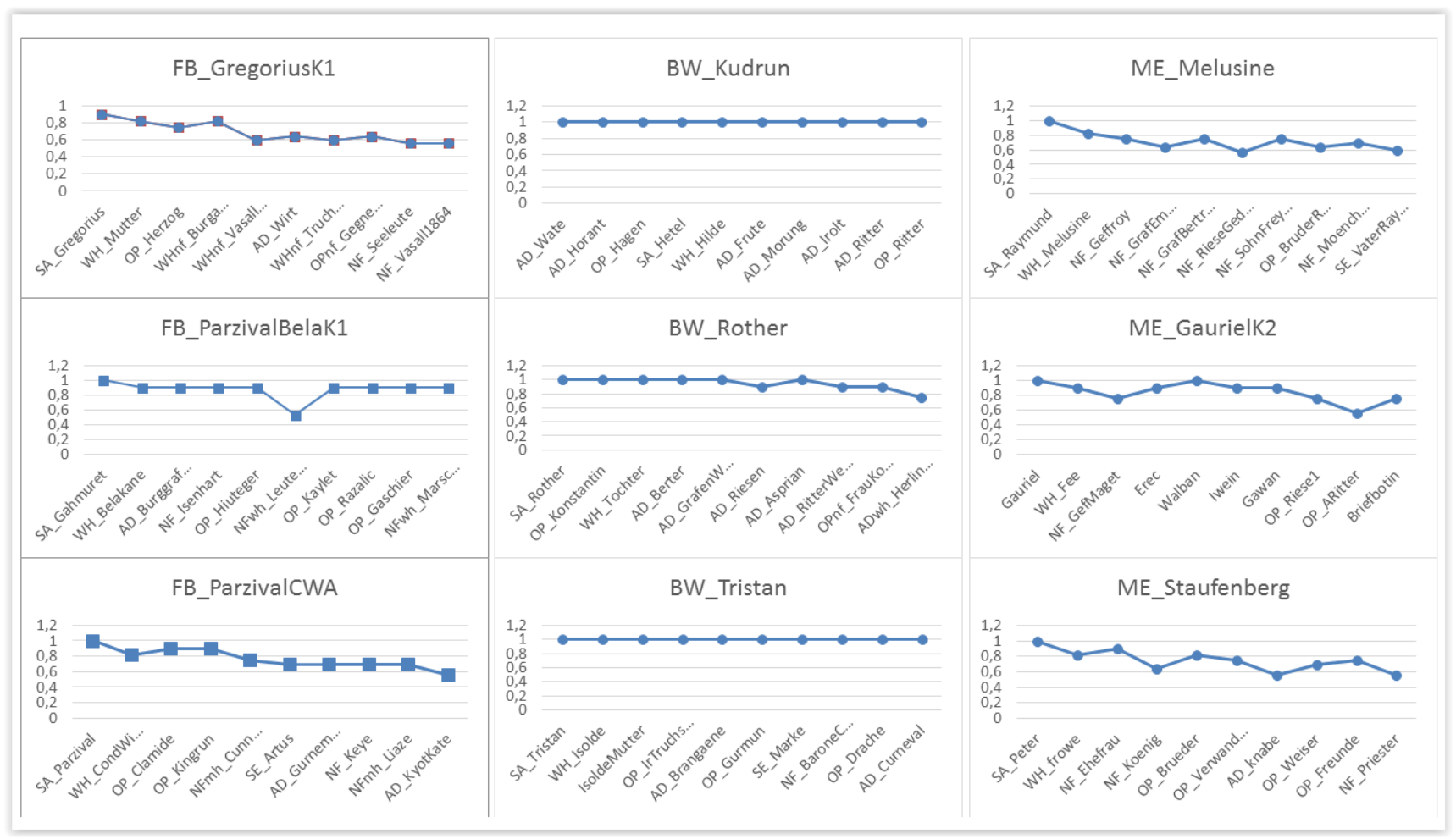

Distanzen: Closeness je Text

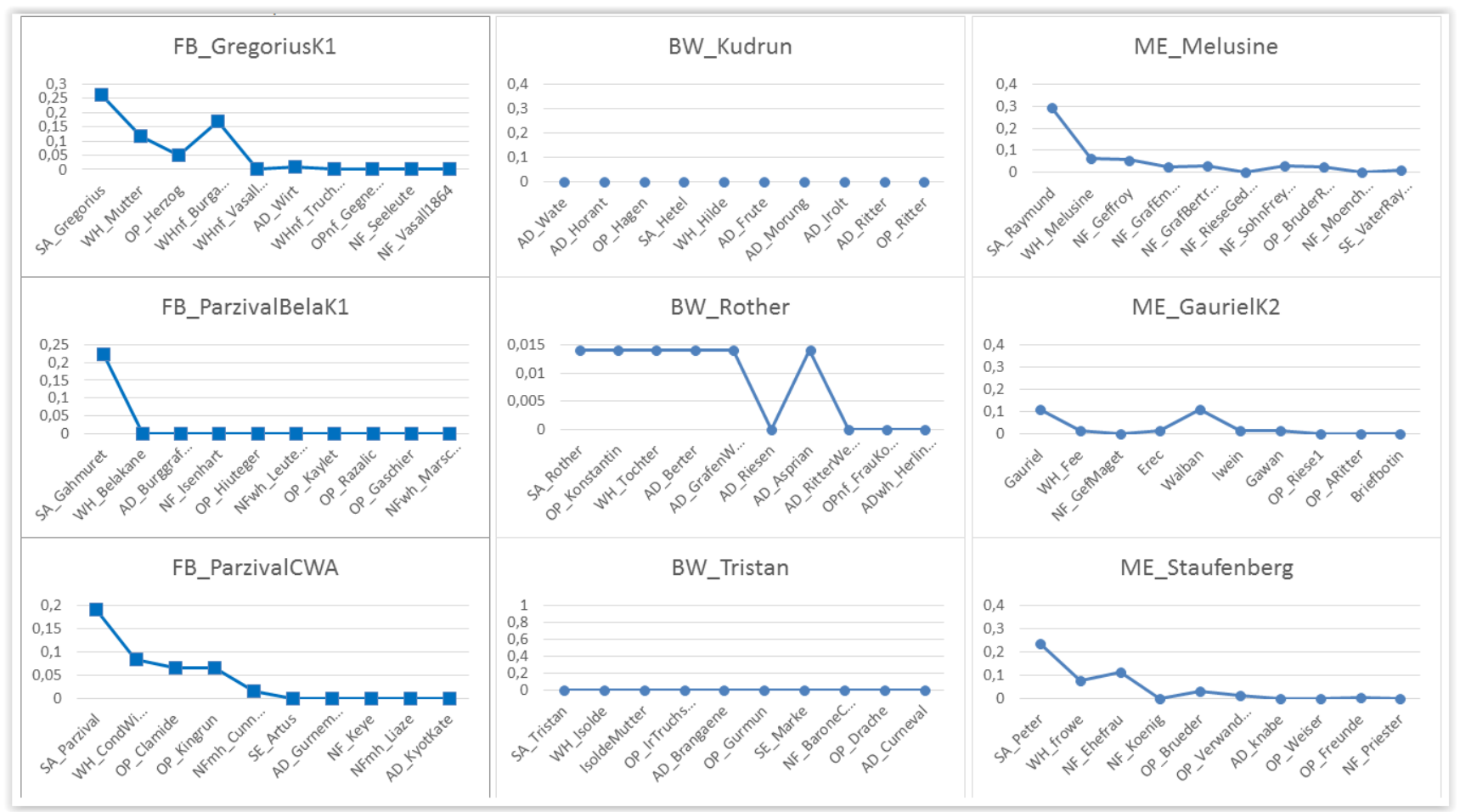

Distanzen: Betweenness je Text 
In den bisherigen Diagrammen wurden die Profile pro Text für die zehn häufigsten Figuren dargestellt. Im Folgenden werden die jeweils Mittelwerte und die Daten für Subjektaktant, weibliche Hauptfigur und Opponent für alle Texte in einem Diagramm erfasst. Die ersten drei Datenpunkte gehören jeweils zu Freier-Befreiungsgeschichten, dann folgen drei Datenpunkte zu Brautwerbungs-Geschichten, die letzten drei gehören zu Mahrtenehe-Erzählungen.

Eine gute Differenzierungsleistung ist dann gegeben, wenn je drei Punkte eines Erzählschemas gleichauf sind und zugleich noch jeweils auf einem anderen Niveau liegen wie die anderen. Das ist jedoch nur manchmal und nur annäherungsweise der Fall.
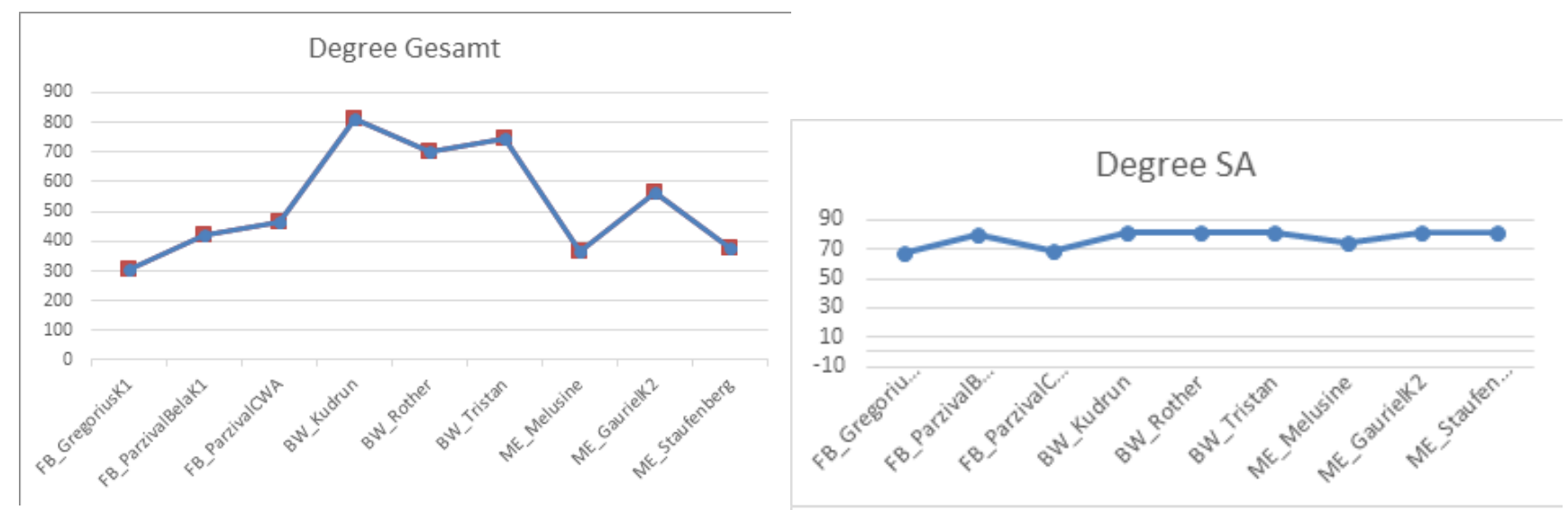

Distanzen: Degree gesamt, SA, WH, OP

Degree WH

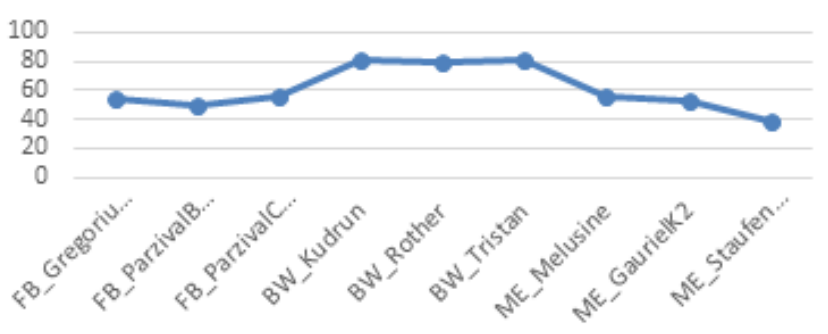

Degree OP Mittelwerte: FB 54,0 BW 77,0 ME 38,3 


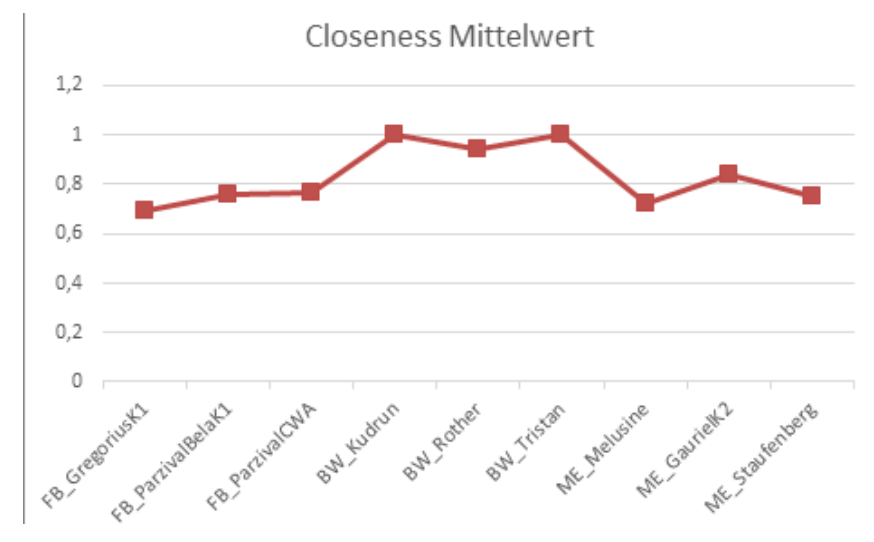

Distanzen: Closeness MW, SA, WH, OP

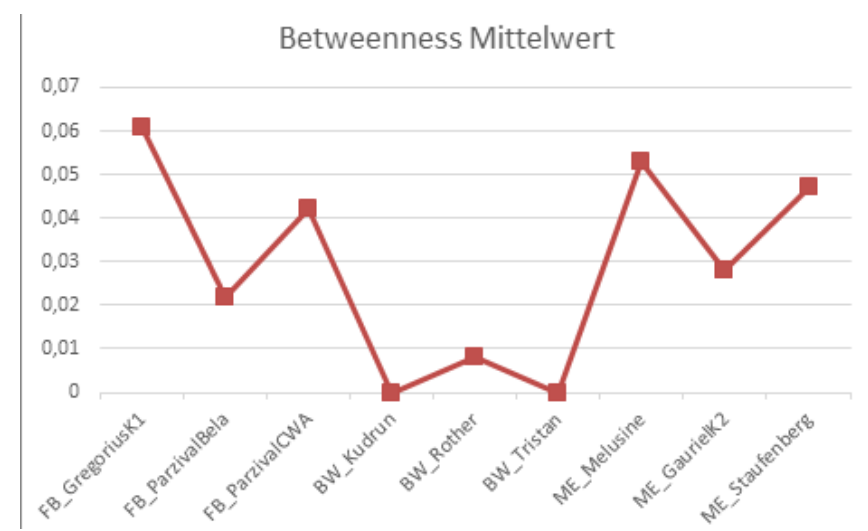

Distanzen: Betweenness MW, SA, WH, OP
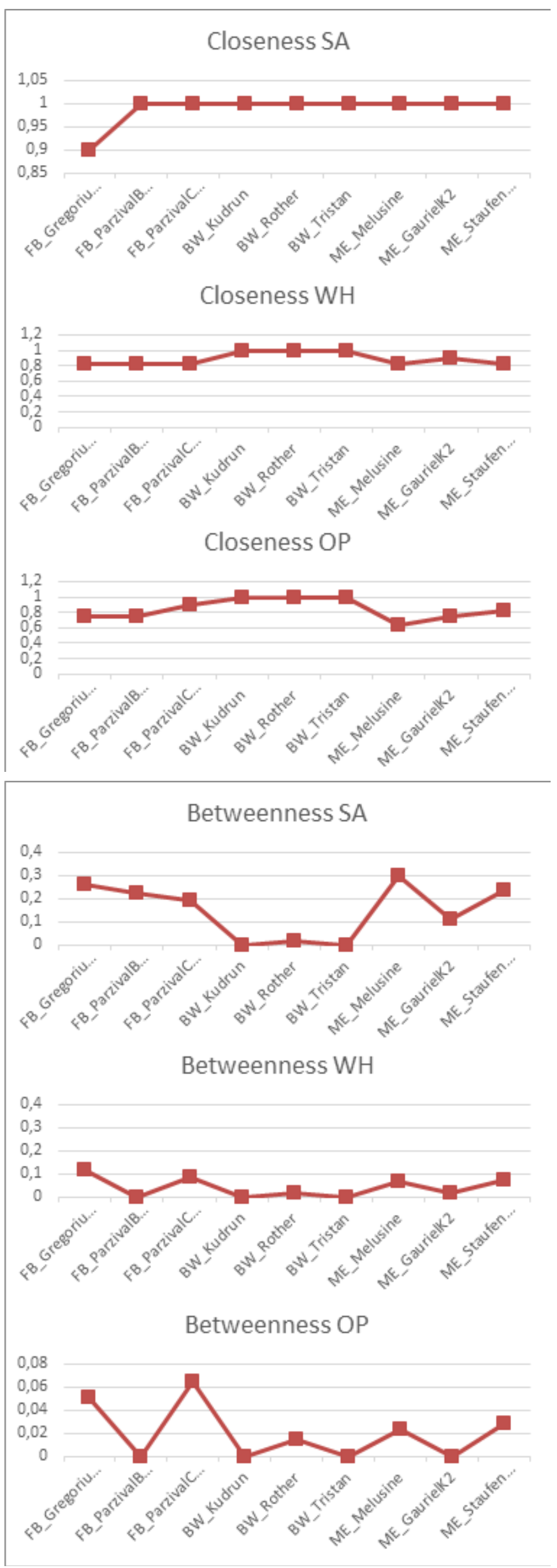


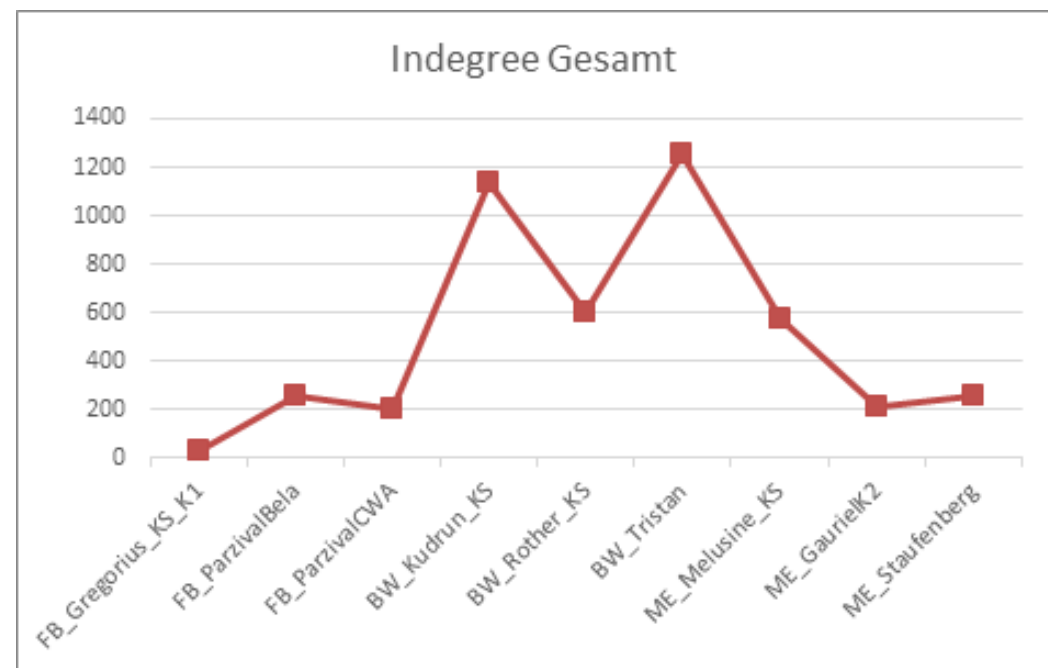

Redewiedergabe: Indegree gesamt, SA, WH, OP

Bei Degree-Werten < 1: Zahl der Referenzen der jeweiligen Redewiedergabe der Figur geteilt durch Gesamtzahl aller Referenzen Redewiedergabe
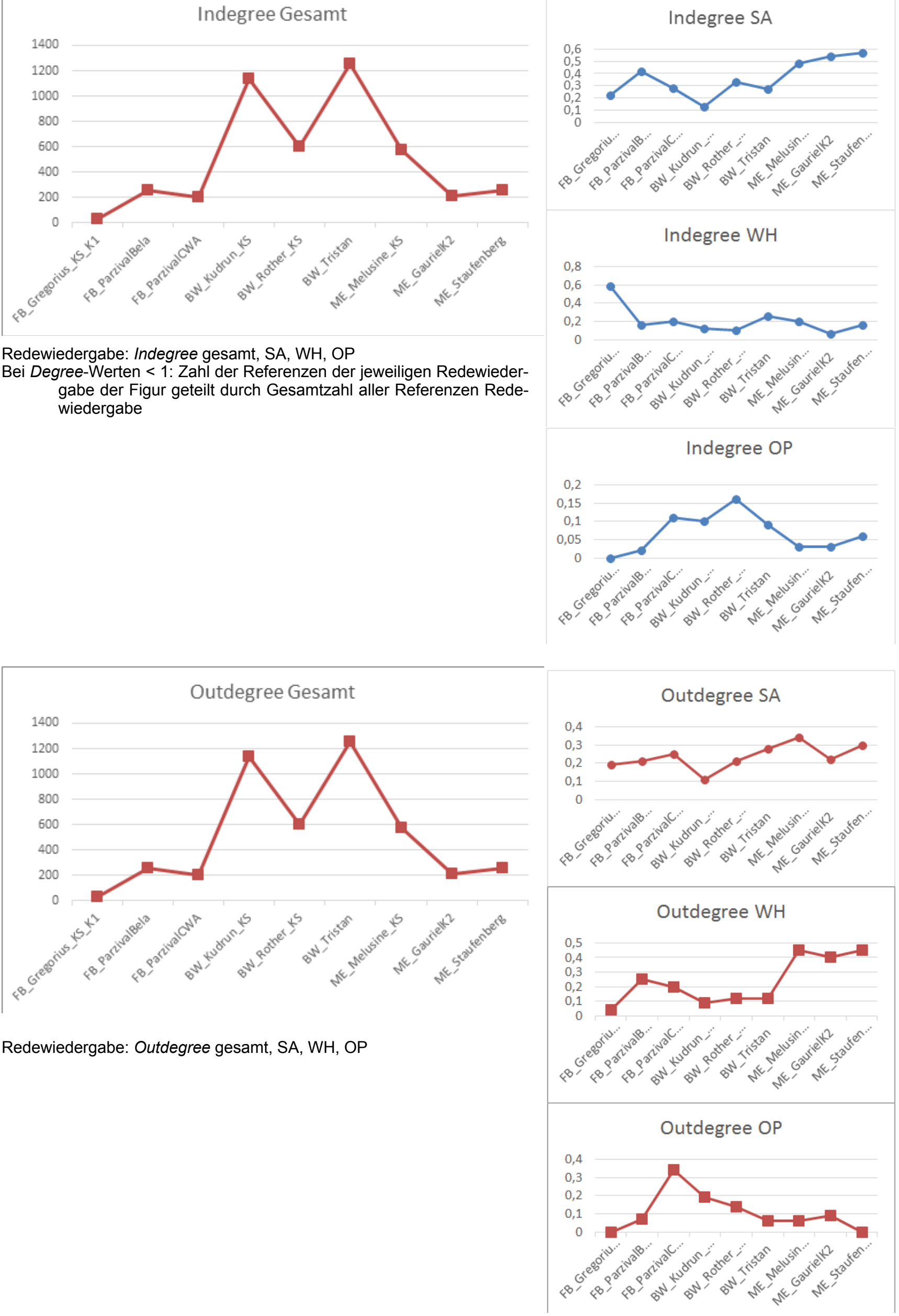
Bei dem UCInet-Network-Centralization-Index kann man besonders anhand der blauen Linie für die Distanzen nochmals die Hauptproblematik erkennen, die sich auch in einigen der vorherigen Diagramme abzeichnet: Das Niveau der jeweiligen Datenpunkte der Erzählschemata wäre dann durchaus verschieden, wenn man je Schema-Erzählung jeweils Durchschnittswerte ansetzen würde. Allerdings gibt es Ausreißer: Der „Staufenberg“ liegt im Bereich, der sonst durch die Freier-Befreiungsgeschichten abgedeckt ist. Spannend wäre es, deutlich mehr Werte zu haben, um dann beurteilen zu können, ob die Mittelwerte je Schema-Erzählung weiterhin differieren und ob die Ausreißerproblematik sich mit mehr Texten als weniger virulent erweist.

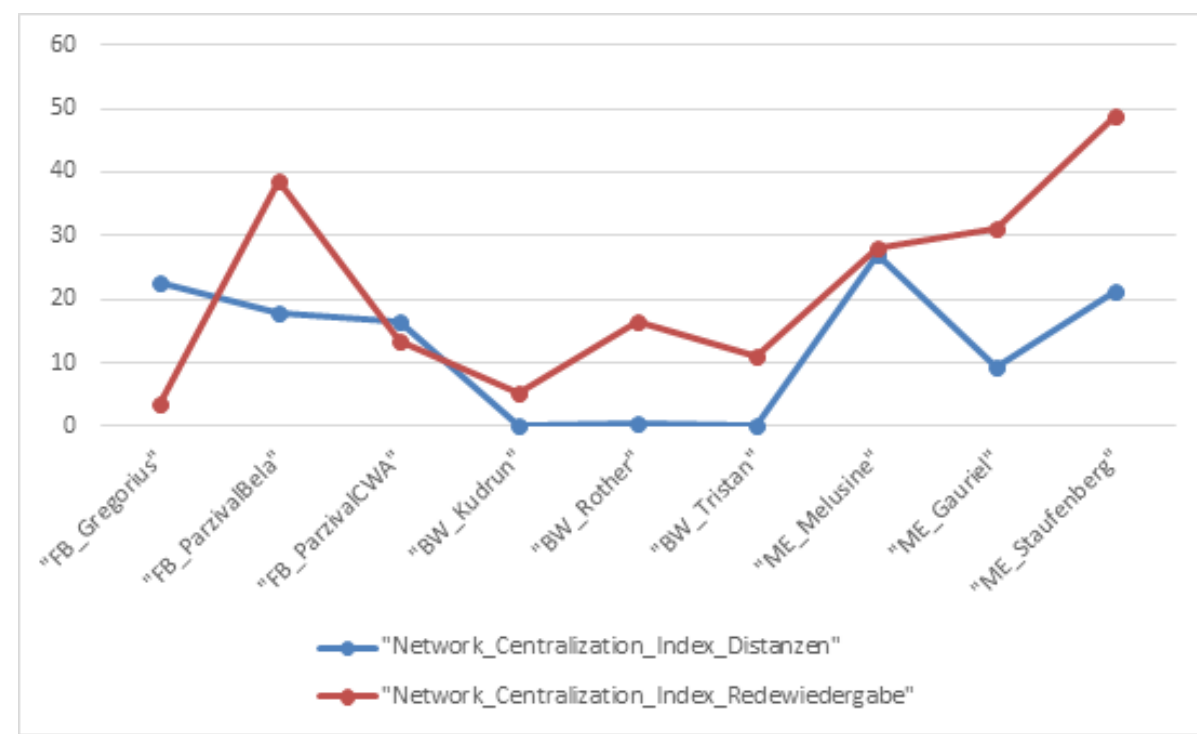

UCInet: Network Centralization Index

\section{Z-Werte und Delta-Plots für alle 29 Zentralitäts-Werte}

Hier werden für alle 29 Zentralitäts-Werte ${ }^{25}$ die jeweiligen Mittelwerte für alle Texte sowie Standardabweichung und Z-Wert berechnet.

Im Diagramm folgt hier der Durchschnitt der Z-Werte für alle Werte. ${ }^{26}$ Hier ist der „Gregorius“-Wert klar als Ausreißer erkennbar.

25 Network Centralization Index Dist, Network Centralization Index Redewiedergabe, Dist MW Degree, Dist MW Closeness, Dist MW Betweenness, Dist Degree SA, Dist Degree WH, Dist Degree OP, Dist Closeness SA, Dist Closeness WH, Dist Closeness OP, Dist Betweenness SA, Dist Betweenness WH, Dist Betweenness OP, ReWie MW Indegree, ReWie MW Outdegree, ReWie Closeness MW, ReWie Indegree SA, ReWie Indegree WH, ReWie Indegree OP, ReWie Outdegree SA, ReWie Outdegree WH, ReWie Outdegree OP, ReWie Closeness SA, ReWie Closeness WH, ReWie Closeness OP, ReWie Betweenness SA, ReWie Betweenness WH, ReWie Betweenness OP.

26 Die Werte repräsentieren die Mittelwerte der Z-Werte in ihrer Summe - anders als bei Burrows' Delta-Berechnung, bei der für jedes Feature einzeln Z-Wert-Differenzen berechnet werden. 


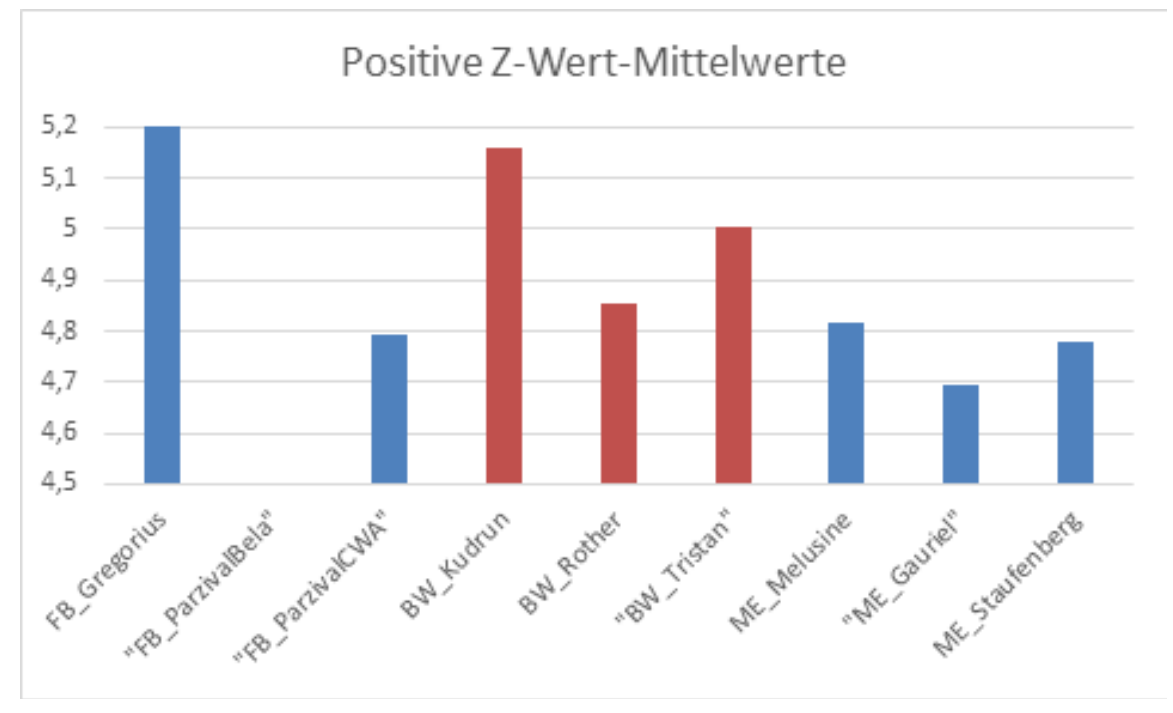

Zu diesen Zentralitäts-Werten lassen sich mit Hilfe von stylo- $R^{27}$ Delta-Distanzen nach Burrows'28 berechnen - siehe Folgeseite. Die auf den ersten Blick erfreuliche Farbsortierung täuscht, denn ,ME_ Gauriel' und ,FB_ParzivalBela' dürften bei einer optimalen Differenzierungsleistung nicht am gleichen Ast hängen. Die Distanz-Tabelle zeigt, dass die Differenzierung bei Brautwerbungs-Erzählungen sehr gut gelingt, Mahrtenehe-Erzählungen werden noch einigermaßen gut separiert, ein Fehler liegt nur beim „Gauriel“, der deutlich zu ,FB_ParzivalBela“ clustert. Die Freier-Befreiungsgeschichten clustern nicht gut.

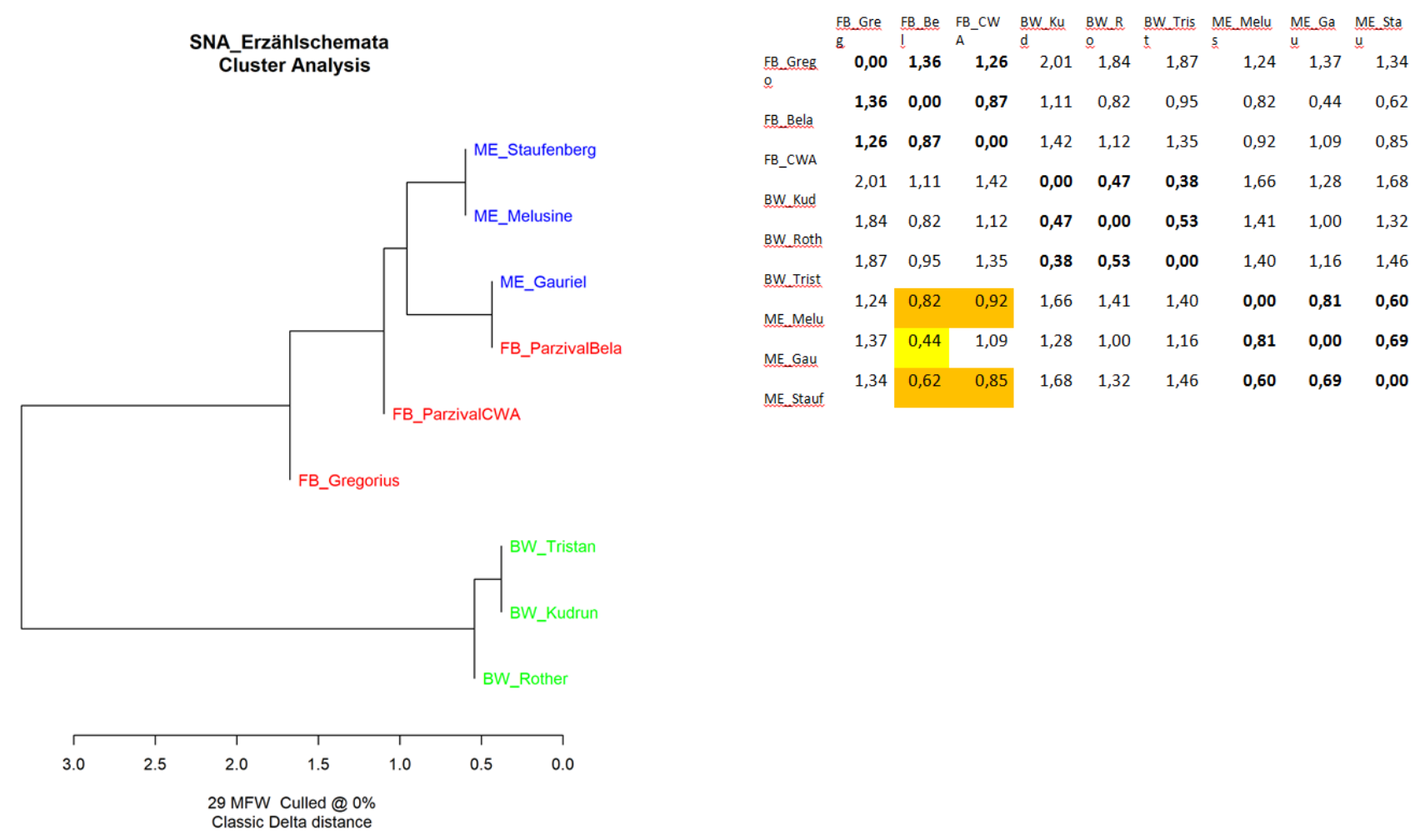

Stylo-R-Delta-Plot zu allen 29 Werten

27 Maciej Eder / Jan RYBICKI / Mike Kestemont, stylo R package, 2017, online unter: https://sites.google.com/site/computationalstylistics/stylo (27.06.2019).

28 John F. Burrows, ,Delta'. A Measure of Stylistic Difference and a Guide to Likely Authorship, in: Literary and Linguistic Computing 17 (2002), 267-287, DOI: 10.1093/Ic/17.3.267. 
Auch wenn man bei den Mahrtenehe-Erzählungen den „Gauriel“ durch Passagen aus „Partonopier und Meliur" ersetzt, wird das Clustering schlechter. Auch hier hat man mit dem Problem zu kalkulieren, dass der Partonopier-Roman nicht nur aus einer Handlung besteht, in der das Mahrtenehe-Schema dominant spürbar wäre, sondern in Teilen kann der Text auch als Minne- und Aventiure-Roman gelten; zudem gibt es Sarazenenkämpfe wie in der Heldenepik. Armin Schulz hat ihn daher als Gattungshybrid beschrieben. ${ }^{29}$ Immerhin grenzt inn die Netzwerkanalyse eindeutig von Brautwerbungs-Erzählungen ab.

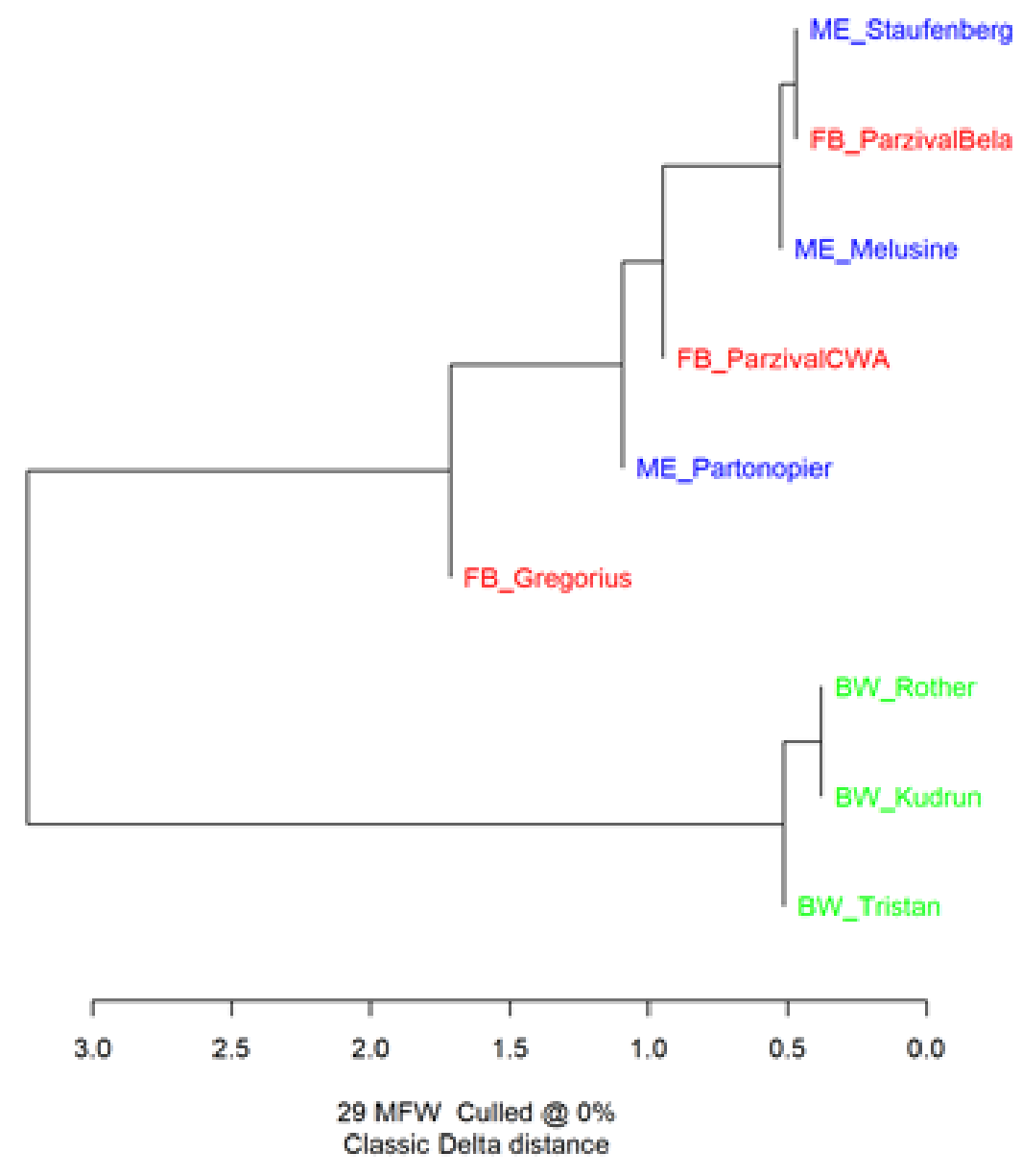

Stylo-R-Delta-Plot zu allen 29 Werten mit ,Partonopier‘ statt ,Gauriel‘

\section{Fazit}

In Bezug auf meine Ausgangsthesen fält die Bilanz positiv aus hinsichtlich der Differenzierung der Brautwerbungs-Erzählungen von anderen Erzählschemata, die Differenzierungsleistung zwischen Freier-Befreiungsgeschichten und Mahrtenehe-Erzählungen bleibt jedoch undeutlich. Als Analyseinstrument für Erzählschemata hat die Netzwerkanalyse also zumindest ein interessantes Potential.

Notwendig wäre nun ein größeres Korpus mit weiteren Texten und weiteren Erzählkernen und Erzählschemata. Möglich wären etwa Muster wie Völkerschlacht/Heidenkrieg, Aventiuren-Schema (costume) in episodisch organisierten Romanen, Brauterwerb durch Tötung des Ehemanns oder des Vaters (Askalon, Lanzelet, Daniel), Brauterwerb im Roman durch Minnedienst (Wigalois, Gawan, ...).

29 Armin Schulz, Poetik des Hybriden. Schema, Variation und intertextuelle Kombinatorik in der Minne- und Aventiureepik: ,Willehalm von Orlens' - ,Partonopier und Meliur - ,Wilhelm von Österreich“ - ,Die schöne Magelone‘, Berlin 2000 (Philologische Studien und Quellen 161), 82-121. 
Zudem könnten Schemavariationen interessant sein - etwa eine Freier-Befreiung ohne Hochzeit, wie man sie im „Iwein“ bei der Dame von Narison findet. Zudem könnte, soweit Texte vorhanden sind, ein Vergleich mit den altfranzösischen Vorlagen und mit späteren Bearbeitungen interessant sein. Anzunehmen wäre, dass sich die netzwerkanalytischen Befunde bei einer ähnlichen Makrostruktur innerhalb von Textreihen als recht homogen erweisen könnten.

Weiterhin wären weitere Experimente mit weiteren Parametern und Kennwerten durchzuführen. Interessant könnte es vor allem sein, Zeitschnitte bei den Zentralitätsmaßen zu erfassen. Wie entwickelt sich etwa die Closeness bei $30 \%$ oder $70 \%$ Handlungsfortschritt bei den verschiedenen Schemata distinkt? Eventuell könnte im Brautwerbungsschema anfangs eine enge Beziehung zwischen Werbungshelfer und Werber evident sein, weniger eng könnte anfangs die Beziehung zur Braut ausfallen. Der letzte Aspekt könnte sich bei Mahrtenehe-Erzählung umgekehrt darstellen - hier wäre zunächst eine starke Fokussierung auf Ritter und Fee zu erwarten.

Offen bleibt vorerst die Frage, ob die Redewiedergabe als Discours-Phänomen repräsentativ für Erzählschemata sein kann. Andere Probleme wie Gattungshybride dürften zentral sein; zudem bleibt das Problem virulent, in welchem Umfang sich ein Text einem Schema zuordnen lässt: Im „Staufenberg“ ist das Mahrtenehe-Schema recht stark spürbar, während sich erhebliche Teile des "Gauriel" hinsichtlich der Figurenkonstellation kaum von anderen nachklassischen Artusromanen unterscheiden. Wichtig wäre also ein Korpus mit besonders typischen Gattungsvertretern.

\section{Literaturverzeichnis}

S.P. Borgatti / M.G. Everett / L.C. Freeman, Ucinet 6 for Windows: Software for Social Network AnalysiHarvard, MA: Analytic Technologies 2002.

Annelen BRUNNER, Automatische Erkennung von Redewiedergabe. Ein Beitrag zur quantitativen Narratologie, Berlin / Boston 2015 (Narratologia 47).

John F. BuRrows, ,Delta'. A Measure of Stylistic Difference and a Guide to Likely Authorship, in: Literary and Linguistic Computing 17 (2002), 267-287, DOI: 10.1093/llc/17.3.267.

Friedrich Michael DIMPEL, Novellenschätze narratologisch auszeichnen und analysieren am Beispiel Victor von Scheffels ,Hugideo‘ und der sozialen Netzwerkanalyse, in: LiLi 47 (2017), 87-108, DOI:10.1007/s41244-0170044-8.

Maciej EdeR / Jan RYBICKI / Mike Kestemont, stylo R package, 2017, online unter: https://sites.google.com/site/ computationalstylistics/stylo (26.06.2019).

Stephan Fuchs, Hybride Helden: Gwigalois und Willehalm. Beiträge zum Heldenbild und zur Poetik des Romans im frühen 13. Jahrhundert, Heidelberg 1997 (Frankfurter Beiträge zur Germanistik).

Algirdas Julien GREIMAS, Strukturale Semantik. Methodologische Untersuchungen. Autorisierte Übersetzung aus dem Französischen von Jens Ihwe, Braunschweig [1966] 1971 (Wissenschaftstheorie, Wissenschaft und Philosophie 4).

Lena Hettinger u. a., Genre classification on German novels, in: 26th International Workshop on Database and Expert Systems Applications (DEXA), 2015, 249-253.

Gert HÜBNER, Erzählform im höfischen Roman. Studien zur Fokalisierung im ,Eneas', im ,Iwein“ und im ,Tristan‘, Tübingen 2003 (Bibliotheca Germanica 44). 
Fotis JANNIDIS, Figur und Person. Beitrag zu einer historischen Narratologie, Berlin / New York 2004 (Narratologia 3).

Dorothea JANSEN, Einführung in die Netzwerkanalyse, Opladen 2003 (UTB 2241).

Benjamin KrautTeR / Janis PAGel, Klassifikation von Titelfiguren in deutschsprachigen Dramen und Evaluation am Beispiel von Lessings ,Emilia Galotti', in: Patrick Sahle, Hg., DHd 2019. Digital Humanities: multimedial \& multimodal. Konferenzabstracts, Köln 2019, 160-164.

Franco MoretTI, Distant Reading. Aus dem Englischen übersetzt von Christine Pries, Konstanz 2016 (2013).

Jan-Dirk MüLler, Höfische Kompromisse. Acht Kapitel zur höfischen Epik um 1200, Tübingen 2007.

Thomas Prolsl u. a., Dependenzbasierte syntaktische Komplexitätsmaße, in: Patrick Sahle, Hg., DHd 2019. Digital Humanities: multimedial \& multimodal. Konferenzabstracts, Köln 2019, 270-273.

Christian ScHMID-CADALBERT, Der ,Ortnit AW“ als Brautwerbungsdichtung. Ein Beitrag zum Verständnis mittelhochdeutscher Schemaliteratur, Bern 1985 (Bibliotheca Germanica 28).

Armin Schulz, Poetik des Hybriden. Schema, Variation und intertextuelle Kombinatorik in der Minne- und Aventiureepik: ,Willehalm von Orlens“ - ,Partonopier und Meliur‘ - ,Wilhelm von Österreich“ - ,Die schöne MageIone', Berlin 2000 (Philologische Studien und Quellen 161).

Armin ScHulz, Erzähltheorie in mediävistischer Perspektive. Herausgegeben von Manuel Braun / Alexandra Dunkel / Jan-Dirk Müller, Berlin / Boston 2012.

Mark Trappmann / Hans J. Hummell / Wolfgang Sodeur, Strukturanalyse sozialer Netzwerke. Konzepte, Modelle, Methoden, Wiesbaden 2005.

Peer TRILCKE, Social Network Analysis (SNA) als Methode einer textempirischen Literaturwissenschaft, in: Philip Ajouri / Katja Mellmann / Christoph Rauen, Hg., Empirie in der Literaturwissenschaft, Münster 2013 (PoetogenesiStudien zur empirischen Anthropologie der Literatur 8), 201-247.

Michael VAUTH, Netzwerkanalyse narrativer Informationsvermittlung in Dramen, in: Patrick Sahle, Hg., DHd 2019. Digital Humanities: multimedial \& multimodal. Konferenzabstracts, Köln 2019, 184-188.

Rainer WARNING, Formen narrativer Identitätskonstitution im höfischen Roman, in: Odo Marquard / Karlheinz Stierle, Hg., Identität, München 1979 (Poetik und Hermeneutik 8), 553-589. 